\title{
Contractor view on project cost overruns
}

\author{
Tobias Lutz,University of Vaasa,tobias.lutz@gmx.ch \\ Josu Takala, University of Vaasa, josu.takala@uwasa.fi \\ Emmanuel Ndzibah, University of Vaasa, emmanuel.ndzibah@uwasa.fi
}

\begin{abstract}
Contractors repeatedly face cost overruns in their projects. This study aims to locate the root causes of cost overrun in the projects of the case company. Moreover, improvement areas are proposed for the mitigation of future cost overruns. To do so, several theories and methodologies are applied: The RAL concept, the Analytical Hierarchy Process, the Critical Factor Indexes, the Sense and Respond methodology, the Manufacturing Strategy Index, the Sustainable Competitive Advantage method, and Knowledge and Technology. Two questionnaires were used for the data collection and were answered by a total of 18 respondents. Challenges in resource management and the cooperation with the client were found to be the root causes of cost overrun in the examined projects. To mitigate future cost overruns, it was proposed to lay a special focus on Resource Management, Project Scheduling, Basic Design, Detailed Design, and Off-site Validation. The Knowledge and Technology results indicate that the products and services of the case company are in the maturity phase of the technology life cycle. Thus, a reduction in production costs is suggested. The highest uncertainty is related to core technology, hence investments in core technology will further contribute to the success of future projects.
\end{abstract}

Keywords: Cost Overrun, Project Management, Knowledge Management, Technology Management, Sense and Respond Method

\section{Introduction}

Project costs tend to overrun. There are numerous famous projects with cost overrun, and worryingly, cost overruns don't seem to be smaller than 100 years ago (Flyvbjerg et al., 2002). Sovacool et al. (2014) assessed the cost related to 401 electricity infrastructure projects in 57 countries. Cost overruns occurred in $75.1 \%$ of the projects with a total of almost $\$ 388$ billion in cost overruns. In the literature, there are diverse explanations why cost overruns occur. Various studies argue that the explanations can either be of technical- or behavioral nature. In the technical category, the actors in a project are assumed to evaluate information and make decisions based on rational reasoning (Segelod, 2018, p. 59). The cost overruns therefore result because of problems predicting the future and are considered "honest" errors. This category also covers all operative inefficiencies during planning and implementation of projects, which lead to higher costs than expected (Cantarelli et al., 2010, pp. 11-12). The behavioral category suggests that emotions drive decision-making and have at least a decisive influence (Segelod, 2018, p. 59). This category explains cost overruns with human bias such as the optimism bias, planning fallacy, and strategic misrepresentation, during project cost estimation (Flyvbjerg et al., 2018, pp. 183-184). The literature usually examines cost overruns from the perspective of the project owners, who must estimate the cost of the whole project to make an investment decision. However, not just project owners, but also 


\section{Issues in Information Systems}

Volume 22, Issue 1, pp. 215-226, 2021

the contractors, hired to operate the projects, struggle with cost overruns. This paper examines the problem of recurring cost overruns from the perspective of a contractor, which participates in competitive tenders.

In competitive tenders, contractors are challenged to make a balanced offer in which the cost estimate must be low enough to offer an attractive bid price and, therefore, a good chance of winning the contract. At the same time, the offer should be high enough to cover possible risks and thereby obviate significant losses (Sonmez et al., 2007). The bid price is the sum of estimated cost and the markup. When the price competition is intense, the costs of the contractors become similar and the chance of further reducing the cost is low. The primary aspect of winning the tender is to set an appropriate markup, which covers the risks arising from project complexity, type of work, location etc. (Jaskowski \& Czarnigowska, 2019). This paper focuses on the technical explanations of cost overrun. Thus, the aim is to ensure operative excellence throughout planning and implementation of the projects and enable an optimal response to risks and errors occurring because of problems predicting the future. This secures that expected profits, anticipated while setting the size of the markup, can be reached.

This paper was conducted in cooperation with a contractor participating in competitive tenders to get new projects awarded. The case company is a global technology company, which plans and delivers electricity-, automation-, instrumentation-, and supervision systems as turnkey projects. The projects provide individual solutions for the clients and usually include engineering, manufacturing, and installation at the customer's site (Personal communication, June 25, 2020). Cost overruns keep occurring in some of these projects. This paper analyses three completed projects in which cost overrun occurred to find appropriate actions to mitigate cost overrun in future projects. This study focuses on the Engineering Process and the Site Management Process of the projects, which contribute the most to the cost overruns of the case company. Shortcomings from these past projects shall not be repeated, and critical processes shall be improved. This leads to the following research question and objectives for this study:

"What are the root causes of cost overrun in projects, and how can this be improved?"

Objective 1: To identify the root causes of cost overrun in the selected projects

Objective 2: To identify areas for improvement to help mitigate cost overrun in the future

\section{Literature review}

This paper combines several theories and methodologies to locate the root causes of cost overrun in the examined projects. The methodologies comprise the RAL concept, the Analytical Hierarchy Process, the Critical Factor Indexes, the Sense and Respond methodology, the Manufacturing Strategy Index, the Sustainable Competitive Advantage method, and Knowledge and Technology. These theories and methodologies can be applied for wide range of research. Similar approaches were amongst others used by Heimonen \& Takala (2017) and Tilabi et al. (2019) to optimize company resources to achieve sustainable competitive advantage. Further goals of these studies were to improve the operations strategy of the case companies and to assist with the decision-making process related to technology and knowledge.

\section{Operations Strategy}

A company's operations are the resources that create and deliver products and services based on customer requests. A company sets the role, objectives, and activities of its processes in the operations strategy (Slack et al., 2013, p. 70). The resources are allocated based on how the company competes in the market 


\section{Issues in Information Systems}

Volume 22, Issue 1, pp. 215-226, 2021

and how it estimates its business environment. In a successful operations strategy, opportunities are identified and prioritized while being aware of possible trade-offs (Takala, Shylina, et al., 2013, pp. 6566). To follow a successful operations strategy, which fits the organization, is crucial. It contributes to the success of the projects and can, therefore, mitigate cost overruns.

According to Miles et al. (1978), there are four pure forms of strategic types of organizations: Defenders, Analyzers, Prospectors, and Reactors. They all have their configuration of technologies, structures, and processes that are consistent with their market strategy. Even if organizations operate in the same industry, they can fit in different categories of strategic types and therefore differentiate themselves from each other. Defenders focus on cost (Takala et al., 2012). They like to operate in an environment where a stable form of organization is suitable. Therefore, they produce only a limited set of products directed at a narrow segment of the total potential market. They react aggressively to prevent competitors from entering their niche market (Miles et al., pp. 550-551). Prospectors focus on quality (Takala et al., 2012). Compared to the Defenders, the Prospectors have an oppositional approach of reacting to the chosen environment. Their prime capability is to find and exploit new product and market opportunities and therefore maintain a reputation as an innovator in product and market development (Miles et al., 1978, pp. 551-553). The focus of the Analyzer type is on balancing quality, cost, and time (Takala et al., 2012). The Analyzer is a combination of the Defender and Prospector types and can be a reasonable alternative to these other strategies. An Analyzer tries to minimize risk while maximizing the opportunity for profit (Miles et al., 1978, pp. 553-557). The strategy types described above are all proactive to the environment in their way. The Reactor, however, adjusts to its environment in both an inconsistent and unstable way. Reactors keep reacting inappropriately to environmental change and uncertainty, which leads to poor outcomes. The Reactor strategy is a result of improperly pursuing one of the other three strategies (Miles et al., 1978, pp. 557-558). Which operations strategy to pursuit must be primarily based on market needs and requirements (Takala, Shylina, et al., 2013, p. 66). The available resources of a company must be aligned to the chosen strategy type, which enables to exploit its advantages as good as possible.

The key which strategy type to choose in the decision-making process of operations managers is the competitive priorities. They indicate the strategic emphasis on developing individual capabilities, which improve the market position of a company. The Responsiveness, Agility, and Leanness (RAL) model is a holistic and multi-focused operations strategy tool based on business goals. The main components of the RAL model are Quality, Cost, Time and Flexibility, which are the most important competitive priorities. They can be used to compare all types of companies with each other (Takala et al., 2012). To get a ranking of the RAL components, and therefore an overview of the competitive priorities of the company, the Analytical Hierarchy Process (AHP) is used. By ranking the components, it can be seen which preference has been given to which component (Saaty, 2008, p. 84). The priorities of the components allow us to analyze if the resources of the projects have been appropriately allocated.

\section{Sense and Respond Methodology}

The Sense and Respond (S\&R) Methodology was introduced to develop the operative management system (Liu \& Takala, 2012). It helps companies to understand their business situation better and therefore enables a fast and more precise reaction to shortcomings (Takala, Muhos, et al., 2013, p. 62). The S\&R method "senses" the critical processes of the operations management, which allows developing them effectively. This approach leads to a competitive advantage (Takala, Shylina, et al., 2013, p. 65).

The S\&R method can be applied as a questionnaire. The questionnaire defines attributes that represent market needs. It enables to react to the present important attributes in a way that the attributes can be developed and changed in the right direction (Takala, Shylina, et al., 2013, p. 67). The respondent must 


\section{Issues in Information Systems}

Volume 22, Issue 1, pp. 215-226, 2021

evaluate the expectation and experience of every performance attribute. The evaluation is on a scale of 1 to 10 in the defined duration. Furthermore, the direction of development of the attribute must be determined on a scale of "worse", "same", or "better". For the analysis, the counts of "better" and "worse" are derived into percentage weights. Several indexes were introduced to evaluate the S\&R questionnaire. This study uses the Normalized Scaled Critical Factor Index (NSCFI), created by Liu and Liang (2015). This index can be used to optimize strategic adjustments and therefore support the faststrategic decision-making process (Liu \& Liang, 2015, pp. 1026-127).

The results of the questionnaire calculated by the NSCFI can be illustrated in a bar chart. The bars are in the colors of a traffic light. Red attributes are under-resourced and therefore critical. They must be thoroughly examined, and additional resources should be attributed to them (Takala, Shylina, et al., 2013, p. 87). Green attributes are balanced. Yellow attributes are over-resourced, and it must be considered if these attributes can be allocated more effectively. An equally distributed resource allocation is considered to be ideal (Takala, Koskinen, et al., 2013, p. 48). The average resource level is defined by dividing the whole resource, which is $100 \%$, to the total number of attributes. The upper bound of a balanced attribute is calculated by adding $1 / 3$ of the average resource level to the average resource level. The lower bound is found when $1 / 3$ of the average resource level is subtracted from the average resource level. CFI values below the lower bound are defined as under-resourced. CFIs that are higher than the upper bound are determined to be over-resourced (Liu \& Takala, 2012, pp. 29-30). The method is an excellent indicator to understand which attributes require special attention. The assumption in this study is that by allocating more resources to the critical attributes and therefore improving them, the cost overrun can be mitigated. Cost overruns occur directly or indirectly because of the critical performance attributes, indicated in red.

The strategic types defined by Miles et al. (1978) are integrated into the Sense and Respond methodology by assigning one of the dimensions (Quality, Cost, Time, or Flexibility) of the RAL model to each of the performance attributes. The RAL dimension is chosen for the performance attribute, which represents its focus best (Takala, Koskinen, et al., 2013, p. 46). This approach is used in this study to determine the operations strategy of each project, using the Manufacturing Strategy Index, which will be described in the next section.

\section{Sustainable Competitive Advantage (SCA)}

The Sustainable Competitive Advantage (SCA) method assures that the resources of the company are operating according to the company's strategy (Takala, Muhos, et al., 2013, p. 62). Besides, the method offers a possibility to see which strategy type may bring a better business performance to the company. It is also a way to check whether all units follow the general strategy if the method is used to analyze several branches of a company (Takala, Liu, et al., 2013, p. 1243). The same applies when different projects of a company are examined, as in this study.

The Manufacturing Strategy Index (MSI) is an integrated part of calculating the SCA values and systematically links up manufacturing with corporate strategy. The model enables to evaluate the competitive priorities of the company, to reach competitive advantages in the market. The evaluation allows a classification of the company or examined unit into one of the strategy types, Analyzer, Defender, Prospector, or Reactor (Takala, Shylina, et al., 2013, p. 68). The MSI is modeled based on the multi-criteria priority weights of the four main competitive priorities Quality (Q), Cost (C), Time (T), and Flexibility (F). These are determined by using the AHP method and presented in a function as MSI= f_MSI (Q,C,T,F) (Takala et al., 2012). There is one equation for each group (Prospector, Analyzer, Defender) for the MSI of operational competitiveness (Takala, Koskinen, et al., 2013, p. 49). The closer the MSI-value is to 1, the more significant are the characteristics of the strategy type (Takala et al., 2012). 


\section{Issues in Information Systems}

Volume 22, Issue 1, pp. 215-226, 2021

Finally, the MSI is used to calculate the SCA. The SCA value indicates how well the resource allocation supports the company's operations strategy and is, therefore, a risk measurement tool, which estimates the operations strategy's functionality. The SCA is a value between 0 and 1 . The closer the SCA value is to 1 , the more consistent are the resource allocation and the operations strategy of the company. There are three methods to calculate the SCA: MAPE, RMSE, and MAD (Takala, Koskinen, et al., 2013, pp. 4950). If the SCA is 0.97 or greater, the value is high, and therefore the resource allocation supports the company's operations strategy well. Values between 0.90 and 0.97 are medium-high, and values below 0.90 are low (Takala et al., 2014, p. 72).

\section{Knowledge and Technology $(K / T)$}

The Knowledge and Technology $(\mathrm{K} / \mathrm{T})$ model allows to examine the problem of cost overrun from the perspective of the technologies used in the operations of the projects. The technologies applied in a company can be situated in a technology pyramid and divided into basic-, core-, and spearhead technology. Thereby, it is possible to get a snapshot of the current technological situation of a company. Basic technology illustrates the crucial know-how on which the products and the business are based. They are essential to the business. Core technologies represent the differentiative and sophisticated know-how, which aim to give the company a competitive advantage. The spearhead technologies illustrate the future areas of know-how and are, therefore, the key factors to future markets and businesses (Tuominen et al., 2004, p. 10). For example, in the automotive industry, a car's engine would be basic technology. It is an essential part of a vehicle. Environmental responsibility is the core technology, as it can achieve a competitive advantage in the present. The self-driving car is spearhead technology, as it could be an exciting business opportunity in the future (Heimonen \& Takala, 2017, p. 194). The objective is to locate the type of technology (basic, core, or spearhead), which causes the highest amount of uncertainty for the case company. This procedure allows them to take measures by investing in the technology category, thus reduce the risk and sustain competitive advantage and success in the market (Tilabi et al., 2019, p. 133). The sectors of the technology pyramid can be linked to the technology life cycle, as can be seen in Figure 1 (Tuominen et al., 2004, p. 10).

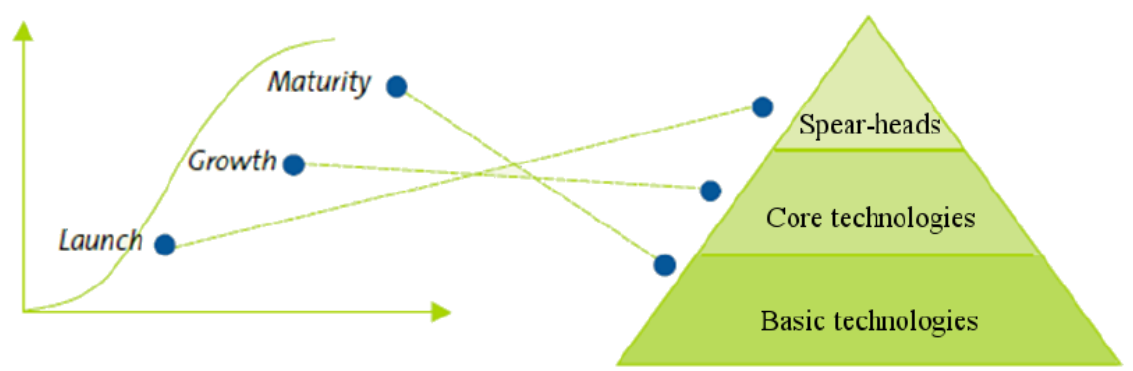

Figure 1. The linkage between the technology life cycle and technology pyramid (Tuominen et al., 2004, p. 10)

When a new product or service is launched, this is closely connected to spearhead technology. In the launching phase, the manufacturing costs are high. In the growth phase, the new product or service has proven itself to be able to make a difference. The production processes are optimized in a way that the product can be produced economically. In this phase, both product and process development are at an optimal level, and core technology is dominating. The maturity phase supersedes the growth phase. In the maturity phase, neither the product nor the processes can be developed much further. This phase is related to basic technology (Tilabi et al., 2019, pp. 138-139). In the technology life cycle, the y-axis stands for the growing technology performance or the cumulative adoption of technology. The willingness of customers to pay for better technology performance sinks, once the performance has reached an 


\section{Issues in Information Systems}

Volume 22, Issue 1, pp. 215-226, 2021

acceptable level. The closer a product or service gets to the maturity phase, the more important it gets to present a balanced offer between price and technology performance. Once the maturity phase is reached, a new disruptive technology might eventually emerge and replace the old one (Adner, 2004, as cited in Taylor \& Taylor, 2012, p. 545). To sustain its position in the market in the maturity phase, a company should lay the focus on reducing the production costs, on newly invented products, and on innovative technology (Tilabi et al., 2019, pp. 138-139).

To determine the technology shares for each performance attribute, a K/T section is added to the S\&R questionnaire. The respondent must divide the technologies used for every performance attribute on a percentage basis in basic-, core-, and spearhead technology. The total share of technology used must equal 100\% (Takala \& Tilabi, 2010, p. 3). The K/T-Data can be analyzed by comparing the NSCFI values to the NSCFI K/T values. The NSCFI K/T values show the resource allocation from the $\mathrm{K} / \mathrm{T}$ perspective (Takala, Koskinen, et al., 2013, p. 48).

The uncertainty regarding each type of technology is calculated by the coefficient of variance (CV), which shows the deviation among the respondents' answers (Takala \& Tilabi, 2010, p. 4). The calculation is based on the assumption that the difference in the opinions of the respondents on the technology shares is the primary source of uncertainty and risk (Tilabi et al., 2019, p. 133). If there is a deviation, there is uncertainty how to use technology to succeed. As a next step, the variability coefficient (VarC) is calculated, which additionally takes the four components of the RAL model into account. Finally, the total amount of risk of technology is calculated, which shows how much the competitiveness of the company falls if the K/T risk materializes. High technology risk levels compromise the success of the projects and therefore lead to cost overrun. A risk of over $100 \%$ questions the whole evaluation based on the criteria (Takala et al., 2016, p. 847).

\section{Empirical research}

Two questionnaires were created for data collection: The S\&R Questionnaire and the AHP Questionnaire. For the S\&R questionnaire, 46 performance attributes were created, which reflect the main operations within the Engineering Process and the Site Management Process of the case company. They can be seen in Table 1. The questionnaires are identical for all three projects, which ensures the comparability. The respondents of the S\&R questionnaire were all experts actively involved in the projects. Answers were received from a total of 15 respondents. The number of answers per attribute varies from 2-5, as not all the respondents could necessarily assess all the performance attributes. The AHP questionnaire is to determine the relative importance of the RAL dimensions and therefore reveal the operations strategy of the case company. The AHP questionnaire was sent to four respondents who are qualified to rank the competitive priorities of the case company. Once the data is analyzed, feedback is needed to uncover the root causes of critical performance attributes and technological uncertainty. Therefore, some respondents were selected for an additional interview.

When examining the competitive priorities of the case company according to the results of the AHP questionnaire, the respondents on average rank Quality $(33,7 \%)$, Cost $(30,5 \%)$, and Time $(28,7 \%)$, as similarly important. Flexibility $(7,2 \%)$ was considered the least essential RAL component. The operations strategy based on MSI tends to be Analyzer, with an MSI-value of 0,978. 
Table 1. Performance attributes of the Sense and Respond questionnaire

\begin{tabular}{|c|c|c|c|c|c|c|c|}
\hline \multicolumn{4}{|c|}{ Supporting Processes } & \multicolumn{4}{|c|}{ Site Management Process } \\
\hline \multirow{2}{*}{ Startup } & 1 & Project scheduling & \multirow{3}{*}{$\begin{array}{l}\leftarrow \text { Time } \\
\leftarrow \text { Quality } \\
\leftarrow \text { Flexibility }\end{array}$} & & 30 & Resource Management & \multirow{17}{*}{$\begin{array}{l}\leftarrow \text { Flexibility } \\
\leftarrow \text { Time } \\
\leftarrow \text { Time } \\
\leftarrow \text { Quality } \\
\leftarrow \text { Time } \\
\leftarrow \text { Quality } \\
\leftarrow \text { Time } \\
\leftarrow \text { Quality } \\
\leftarrow \text { cost }\end{array}$} \\
\hline & 2 & Establishing of project team & & & 31 & Site Establishment and Mobilization & \\
\hline \multirow{14}{*}{ Execution } & 3 & Resource Management & & \multirow{2}{*}{ General } & 32 & Construction and Installation & \\
\hline & 4 & Financial \& Cost Management & $\leftarrow$ Cost & & 33 & Commissioning & \\
\hline & 5 & Communications Management & \multirow{6}{*}{$\begin{array}{l}\leftarrow \text { Quality } \\
\leftarrow \text { cost } \\
\leftarrow \text { cost } \\
\leftarrow \text { cost } \\
\leftarrow \text { Time } \\
\leftarrow \text { cost }\end{array}$} & \multirow{2}{*}{ Automation } & 34 & Construction and Installation & \\
\hline & 6 & Procurement \& Contractor Management & & & 35 & Commissioning & \\
\hline & 7 & Change Management & & \multirow{2}{*}{ Electrical } & 36 & Construction and Installation & \\
\hline & 8 & Risk \& Opportunity Management & & & 37 & Commissioning & \\
\hline & 9 & Schedule Management & & & 38 & Demobilization & \\
\hline & 10 & HSE Management & & & & & \\
\hline & 11 & Quality Management & \multirow{7}{*}{$\begin{array}{l}\leftarrow \text { Quality } \\
\leftarrow \text { Quality } \\
\leftarrow \text { Time } \\
\leftarrow \text { Time } \\
\leftarrow \text { Quality } \\
\leftarrow \text { Quality } \\
\leftarrow \text { cost }\end{array}$} & & & & \\
\hline & 12 & Requirement Management & & & & & \\
\hline & 13 & Decision-making & & & & & \\
\hline & 14 & Information Management & & & & & \\
\hline & 15 & Knowledge Management & & & & & \\
\hline & 16 & Technology Management & & & & & \\
\hline Closeout & 17 & Project acceptance, take-over and closeout & & & & & \\
\hline \multicolumn{4}{|c|}{ Engineering and Validation Process } & \multicolumn{3}{|l|}{ Soft factors } & \\
\hline \multirow{6}{*}{ Automation } & 18 & Resource Management & \multirow{12}{*}{$\begin{array}{l}\leftarrow \text { Flexibility } \\
\leftarrow \text { cost } \\
\leftarrow \text { Quality } \\
\leftarrow \text { Quality } \\
\leftarrow \text { Time } \\
\leftarrow \text { Quality } \\
\leftarrow \text { Flexibility } \\
\leftarrow \text { cost } \\
\leftarrow \text { Quality } \\
\leftarrow \text { Quality } \\
\leftarrow \text { Time } \\
\leftarrow \text { Quality }\end{array}$} & & 39 & Professional relationship & \multirow{12}{*}{$\begin{array}{l}\leftarrow \text { Flexibility } \\
\leftarrow \text { Cost } \\
\leftarrow \text { Quality } \\
\leftarrow \text { Cost } \\
\leftarrow \text { Quality } \\
\leftarrow \text { Cost } \\
\leftarrow \text { Time } \\
\leftarrow \text { Quality }\end{array}$} \\
\hline & 19 & Conceptual Design & & & 40 & Know-How / Experience & \\
\hline & 20 & Basic Design & & & 41 & Knowledge & \\
\hline & 21 & Detailed Design & & & 42 & Competence & \\
\hline & 22 & Manufacturing & & & 43 & Engagement & \\
\hline & 23 & Off-site Validation & & & 44 & Innovation & \\
\hline \multirow{6}{*}{ Electrical } & 24 & Resource Management & & & 45 & Teamwork & \\
\hline & 25 & Conceptual Design & & & 46 & Organizational culture & \\
\hline & 26 & Basic Design & & & & & \\
\hline & 27 & Detailed Design & & & & & \\
\hline & 28 & Manufacturing & & & & & \\
\hline & 29 & Off-site Validation & & & & & \\
\hline
\end{tabular}

The results of the S\&R questionnaire are presented next. When looking at the resource allocation in Project 1 based on NSCFI, the average resource level is at 0,021 , with the upper bound at 0,029 and the lower bound at 0,015 for balanced resource allocations. There are many critical performance attributes in Project $1(1,3,4,7,8,9,12,13,18,19,21,24,25,27,30,33)$. Notable is that all attributes related to Resource Management are critical $(3,18,24,30)$, which therefore seemed to be a big problem. The fact that Change Management (7), Risk \& Opportunity Management (8), Schedule Management (9), Requirement Management (12), and Decision-making (13) are all critical indicates that it was tough to cope with the challenges encountered in the project.

In Project 2, the average resource level based on NSCFI is at 0,021, with the upper bound at 0,029 and the lower bound at 0,015 for balanced resource allocations. There are 19 critical attributes $(1,5,18,20,21$, $23,24,26,27,29,30,32,34,36,37,38,40,42,46)$. The many critical performance attributes in the Engineering Process and Site Management Process are standing out. Resource Management (3, 18, 24, 30 ) is very critical in Project 2 as well. Only no. 3 is narrowly a balanced attribute. The critical attributes in the Engineering Process besides Resource Management $(18,24)$ are Basic Design $(20,26)$, Detailed Design $(21,27)$, and Off-site Validation $(23,29)$.

The average resource level in Project 3 based on NSCFI is at 0,024, with the upper bound at 0,034 and the lower bound at 0,016 for balanced resource allocations. The resource allocation for attributes no. 31, 32, 34,36 , and 38 cannot be calculated, as they were not part of the case company's scope and are therefore not feasible for the cost overrun of the case company in this project. In Project 3, there are twelve critical 


\section{Issues in Information Systems}

Volume 22, Issue 1, pp. 215-226, 2021

attributes $(9,13,16,17,26,28,29,30,37,40,41,42)$. In this project, Resource Management is only critical in the Site Management Process (30).

When comparing the three projects to each other, There was only one performance attribute, which was critical in all three projects. It was Resource Management in the Site Management Process (30). However, many performance attributes were critical in two of the projects or were only narrowly in the balanced zone. Still, every project had different challenges. However, having the right personnel resources at the right time caused difficulties in all three projects.

Table 2. Average of expectations of all respondents in all three projects (Top 10)

\begin{tabular}{|r|r|l|l|l|r|}
\hline \multicolumn{2}{|l}{ Rank No. Attribute name } & Engineering and Validation Process & Electrical & Avg. expectation \\
\hline $\mathbf{1}$ & $\mathbf{2}$ & Resource Management & Engineering and Validation Process & Automation & $\mathbf{8 , 3 6 4}$ \\
\hline $\mathbf{2}$ & 23 & Off-site Validation & Supporting Processes & Startup & $\mathbf{8 , 3 3 3}$ \\
\hline $\mathbf{3}$ & 1 & Project scheduling & Engineering and Validation Process & Automation & $\mathbf{8 , 2 2 2}$ \\
\hline $\mathbf{3}$ & 18 & Resource Management & Engineering and Validation Process & Automation & $\mathbf{8 , 2 2 2}$ \\
\hline $\mathbf{3}$ & 21 & Detailed Design & Engineering and Validation Process & Electrical & $\mathbf{8 , 2 2 2}$ \\
\hline $\mathbf{3}$ & 26 & Basic Design & Engineering and Validation Process & Automation & $\mathbf{8 , 2 2 2}$ \\
\hline $\mathbf{7}$ & $\mathbf{2}$ & Basic Design & Supporting Processes & Execution & $\mathbf{8 , 1 1 1}$ \\
\hline $\mathbf{8}$ & $\mathbf{3}$ & Resource Management & Engineering and Validation Process & Electrical & $\mathbf{8 , 0 0 0}$ \\
\hline $\mathbf{8}$ & $\mathbf{2 9}$ & Off-site Validation & Site Management Process & - & $\mathbf{8 , 0 0 0}$ \\
\hline $\mathbf{1 0}$ & 30 & Resource Management & Soft factors & - & $\mathbf{7 , 9 1 7}$ \\
\hline $\mathbf{1 0}$ & 42 & Competence & & $\mathbf{7 , 9 1 7}$ \\
\hline
\end{tabular}

Table 2 shows the top ten of the combined average of expectations of all respondents in all three projects. It represents the respondent's opinion on how well the performance attributes must perform in future projects so that the projects stay on budget and no cost overrun occurs. The table is derived from the S\&R questionnaire answers. Resource Management (rank no. 1, 3, 8, 10) is expected to perform very well, with an average expectation of at least 7,917 out of 10. The respondents also have very high expectations (over 8) towards Off-site Validation (attribute 12, 29), Project scheduling (1), Detailed Design (21), and Basic Design $(26,20)$.

The results of the MSIs indicate how well the resource allocation based on the NSCFI-values support the operations strategy of the case company. The strategy type based on NSCFI-values according to Project 1 and Project 2 tends to be Analyzer. This result corresponds with the strategy type of the case company, which tends to be Analyzer as well. The strategy type of Project 3 has a slight tendency to be Prospector, but all calculated values for Project 3 are relatively close to each other.

Table 3. SCA values of the projects

\begin{tabular}{|c|c|c|c|c|c|c|c|}
\hline \multirow{2}{*}{ Project } & MAPE & RMSE & MAD & MAPE & RMSE & MAD \\
\cline { 2 - 7 } & \multicolumn{3}{|c|}{ NSCFI } & \multicolumn{3}{|c|}{ NSCFI K/T } \\
\hline 1 & 0,99 & 1,00 & 1,00 & 0,99 & 0,99 & 0,99 \\
\hline 2 & 0,98 & 0,99 & 0,99 & 0,95 & 0,97 & 0,97 \\
\hline 3 & 0,95 & 0,97 & 0,97 & 0,93 & 0,96 & 0,96 \\
\hline
\end{tabular}

The resource allocation in the projects is compared to the operations strategy of the case company by calculating the SCA values. The results are as in Table 3. In Project 1 and Project 2, MAPE, RMSE, and MAD are very close to 1 , or even 1 , which means the resource allocation, according to NSCFI, is almost entirely supporting the operations strategy of the case company. The values for NSCFI T/K in Project 1 are also very close to 1 . In Project 2 , the NSCFI K/T values are only medium-high, as they are between 


\section{Issues in Information Systems}

Volume 22, Issue 1, pp. 215-226, 2021

0,90 and 0,97 . The SCA values of Project 3 confirm that the resource allocations don't support the operations strategy as well as in Project 1 and Project 2. The values in Project 3 are lower, but the resource allocations support the operation's strategy also well. NSCFI T/K values are slightly lower than the NSCFI values.

When calculating the average technology shares of the three projects combined, the dominance of basic technology with $78 \%$ is striking. Basic technologies are commonly used and should, therefore, be familiar to the employees. The share of spearhead technology is very low (4\%), which means that almost no innovative technologies, which illustrate future areas of know-how, were used. $18 \%$ of the technologies belonged to core technology.

The CV values show that most uncertainty in all three projects is related to core technology, with a value of 6,20 on average. The second most uncertainty is related to spearhead technology $(4,90)$, and the least uncertainty is related to basic technology $(3,84)$.

If the $\mathrm{K} / \mathrm{T}$ risk materialized, the competitiveness of Project 1 might have fallen up to $85 \%$, the competitiveness of Project 2 up to $37 \%$, and the competitiveness of Project 3 up to $40 \%$. The most significant risk according to the $\mathrm{VarC}$ values is related to Quality in all three projects. However, all values are clearly under $100 \%$, which means that the uncertainty levels are passable.

\section{Discussion}

The research question will be answered with the help of the two objectives of this study.

Objective 1: To identify the root causes of cost overrun in the selected projects

In Project 1, according to the respondents, the cost overrun mainly occurred because of too few or wrong personnel resources (Resource Management). Another leading cause was the challenging consultant of the client, who was very demanding. In the interviews, the critical performance attributes of the S\&R analysis could mainly be traced back to these two influencing factors. In Project 2, it was insufficient Resource Management, which was the root cause of the cost overrun. The inadequate resources led to the criticality of most critical performance attributes, which all contributed to the ultimate cost overrun. In Project 3, the situation looks somewhat different. Some challenges concerning Resource Management occurred only in the Site Management Process. The root cause was less exact, but the critical attributes were connected to the fact that the whole project was bureaucratic. There were unclear requirements from the client and his consultant, which led to different understandings.

Objective 2: To identify areas for improvement to help mitigate cost overrun in the future

After having located the root causes of cost overrun in the selected projects, some possible areas for improvement are located. The case company should lay a special focus on the performance attributes with the highest impact on cost overrun, as presented in Table 2. Performance attributes with high average expectation should be improved by allocating more resources, as these are the ones that have the highest impact on cost overrun. Moreover, all attributes with an average expectation of at least 8 were critical in at least one of the projects.

Most performance attributes related to Resource Management were critical in Project 1, and Project 2. In Project 3, Resource Management was critical in the Site Management Process. In the additional interviews, Resource Management was identified as a root cause for cost overrun in Project 1 and Project 


\section{Issues in Information Systems}

Volume 22, Issue 1, pp. 215-226, 2021

2. This result allows the conclusion that cost overrun could be mitigated the best in the future by improving Resource Management. This conclusion is underlined by the fact that all performance attributes connected to Resource Management appear on top in Table 2.

Another factor with a high impact on cost overrun in Project 1 and Project 3 were the consultants of the clients. Also, the client was new in Project 3. The cooperation was bureaucratic and time-consuming in both projects. Special attention should therefore be given to projects implemented for new clients, or where clients involve consultants.

In the technology life cycle, a clear dominance of basic technology and low amounts of core- and spearhead technology is a sign that the products and services offered have reached the maturity phase. Cost reductions are essential for competitive advantage of the case company, as customers are not necessarily ready to pay a premium for better technical performance. High shares of basic technology furthermore mean that the case company can't differentiate from competitors from a technological perspective.

The highest uncertainty in all three projects is related to core technology. It is the technology that should most be invested in to gain and sustain competitive advantage and to succeed in projects. However, spearhead technology should not be neglected. These investments will ultimately contribute to the mitigation of cost overruns.

\section{Conclusions}

This research aimed to locate the root causes of cost overrun from a contractor's perspective and to present improvement proposals for its mitigation. A wide range of methods was used for this purpose to gain a comprehensive picture of the situation in the case company. The methods comprised the Analytical Hierarchy Process (AHP), the Critical Factor Indexes (CFI), the Sense and Respond method (S\&R), the RAL-concept, the Manufacturing Strategy Index (MSI), the Knowledge and Technology method (K/T), and the Sustainable Competitive Advantage model (SCA).

Based on the analysis, it can be concluded that there were mainly two root causes of cost overrun in the examined projects. The first and most serious root cause is Resource Management. The second root cause found was the cooperation with the client and the client's consultant, which was challenging Project 1 and Project 3. The research furthermore presented improvement proposals for the mitigation of cost overrun in future projects.

The data was collected with the help of questionnaires. The methods used could point out which operations were critical in the projects and therefore contributed most to the cost overruns, as they examined the projects from an operative and a technological perspective. The results and the proposed areas for improvement allow addressing the problem of recurring cost overruns specifically.

\section{References}

Cantarelli, C. C., Flyvbjerg, B., Molin, E. J. E., \& van Wee, B. (2010). Cost Overruns in Large-scale Transportation Infrastructure Projects: Explanations and Their Theoretical Embeddedness. European Journal of Transport and Infrastructure Research, 10(1), 5-18.

Flyvbjerg, B., Holm, M. S., \& Buhl, S. (2002). Underestimating costs in public works projects: error or lie? Journal of the American Planning Association, 68(3), 279-295. 


\section{Issues in Information Systems}

Volume 22, Issue 1, pp. 215-226, 2021

Flyvbjerg, B., Ansar, A., Budzier, A., Buhl, S., Cantarelli, C. C., Garbuio, M., Glenting, C., Holm, M. S., Lovallo, D., Lun, D., Mohlin, E., Rønnest, A., Stewart, A., \& van Wee, B. (2018). Five things you should know about cost overrun. Transportation Research Part A, 118, 174-190.

Heimonen, K., \& Takala, J. (2017). Resource Optimization for Sustainable Competitive Advantage in Residential Project Development: Empirical Evidence From a Medium-Sized Enterprise in Finland. In M. Vemic (Eds.), Strategic Optimization of Medium-Sized Enterprises in the Global Market (pp. 185-212). IGI Global.

Jaskowski P., \& Czarnigowska, A. (2019). Contractor's bid pricing strategy: a model with correlation among competitor's prices. Open Engineering, 9(1), 159-166.

Liu, Y., \& Liang, L. (2015). Evaluating and developing resource-based operations strategy for competitive advantage: an exploratory study of Finnish high-tech manufacturing industries. International Journal of Production Research, 53(4), 1019-1037.

Liu, Y., \& Takala, J. (2012). Operations strategy optimization based on developed sense and respond methodology. Risus - Journal on Innovation and Sustainability, 3(1), 25-34.

Miles, R. E., Snow, C. C., Meyer, A. D., \& Coleman, H. J. (1978). Organizational Strategy, Structure and Process. The Academy of Management Review, 3(3), 546-561.

Saaty, T. L. (2008). Decision making with the analytic hierarchy process. International Journal of Services Sciences, 1(1), 83-98.

Segelod, E. (2018). Project Cost Overrun. Causes, Consequences, and Investment Decisions. Cambridge University Press.

Slack, N., Brandon-Jones, A., \& Johnston, R. (2013). Operations Management. 7th Ed. Pearson.

Sonmez, R., Ergin, A., \& Birgonul, T. M. (2007). Quantitative Methodology for Determination of Cost Contingency in International Projects. Journal of Management in Engineering, 23(1), 35-39.

Sovacool, B. K., Gilbert, A., \& Nugent, D. (2014). An international comparative assessment of construction cost overruns for electricity infrastructure. Energy Research \& Social Science, 3, 152-160.

Takala, J., \& Tilabi, S. (2010). Towards Developing a Decision Making Tool for Technology and Knowledge Priorities. Management and Production Engineering Review, 1(2), 10-20.

Takala, J., Kamdee, T., Hirvela, J., \& Kyllönen, S. (2012). Analytical calculation of global operative competitiveness. Paper presented at the 16th International Conference on Management of Technology, Management of Technology for Service Economy, Orlando: International Association for Management of Technology.

Takala, J., Koskinen, J., Liu, Y., Tas, M. S., \& Muhos, M. (2013). Validating Knowledge and Technology Effects to Operative Sustainable Competitive Advantage. Management and Production Engineering Review, 4(3), 45-54. 


\section{Issues in Information Systems}

Volume 22, Issue 1, pp. 215-226, 2021

Takala, J., Liu, Y., Feng, B., \& Yang, W. (2013). Analytical Evaluation of Sustainable Competitive Advantage. IFAC Proceedings Volumes, 1240-1243. Saint Petersburg: International Federation of Automatic Control.

Takala, J., Muhos, M., Tilabi, S., Tas, M. S. \& Yan, B. (2013). Using Sustainable Competitive Advantages to Measure Technological Opportunities. Management and Production Engineering Review, 4(3), 55-64.

Takala, J., Shylina, D., \& Tilabi, S. (2014). How to apply Sustainable Competitive Advantage for Regional Developments (Case: Ostrobothnia Region of Finland). Management and Production Engineering Review, 5(2), 66-77.

Takala, J., Shylina, D., Forss, T., \& Malmi, J. (2013). Study on Resource Allocations for Sustainable Competitive Advantage. Management and Production Engineering Review, 4(3), 65-75.

Takala, J., Zucchetti, P., Daneshpour, H., Kunttu, S., Välisalo, T., Pirttimäki, J., \& Kiiski, P. (2016). The Evaluation of Investment Decision Making with Knowledge \& Technology Rankings and the Sand Cone Model. Proceedings of the 13th International Conference on Innovation \& Management, 846-850. Kuala Lumpur: Wuhan University of Technology Press.

Taylor, M., \& Taylor, A. (2012). The technology life cycle: Conceptualization and managerial implications. International Journal of Production Economics, 140(1), 541-553.

Tilabi, S., Tasmin, R., Takala, J., Palaniappan, R., Hamid, N. A., \& Ngadiman, Y. (2019). Technology development process and managing uncertainties with sustainable competitive advantage approach. Acta logistica, 6(4), 131-140.

Tuominen, T., Rinta-Knuuttila, A., Takala, J., \& Kekäle, T. (2004). Technology survey: logistics and automation branch of materials handling industry. Logistia Magazine, 8-11. 